\title{
Hydrogen-rich saline attenuates chemotherapy-induced ovarian injury via regulation of oxidative stress
}

\author{
XIAOYIN MENG ${ }^{1 *}$, HONGGUANG CHEN ${ }^{2,3 *}$, GUOLIN WANG ${ }^{2,3}$, YONGHAO YU ${ }^{2,3}$ and KELIANG XIE ${ }^{2,3}$ \\ ${ }^{1}$ Department of Gynecology and Obstetrics, Tianjin Hospital; ${ }^{2}$ Department of Anesthesiology, \\ Tianjin Institute of Anesthesiology, Tianjin Medical University General Hospital, \\ Tianjin 300052; ${ }^{3}$ Tianjin Institute of Anesthesiology, Tianjin 300052, P.R. China
}

Received June 8, 2014; Accepted July 31, 2015

DOI: $10.3892 /$ etm.2015.2787

\begin{abstract}
Hydrogen has been reported to exert a therapeutic effect in several diseases due to its antioxidative, anti-inflammatory and anti-apoptotic properties. The aim of the present study was to investigate whether hydrogen-rich saline treatment could attenuate ovarian damage induced by cisplatin. A total of 240 adult, virgin, female Sprague Dawley rats, weighing 180-220 g, were randomly divided into four groups ( $\mathrm{n}=60$ per group): Control (Con), control + hydrogen-rich saline $\left(\mathrm{Con}+\mathrm{H}_{2}\right.$ ), cisplatin-induced ovarian injury (OI) and cisplatin-induced ovarian injury + hydrogen-rich saline $\left(\mathrm{OI}+\mathrm{H}_{2}\right)$. Cisplatin was diluted in saline immediately before use. In the $\mathrm{OI}$ and $\mathrm{OI}+\mathrm{H}_{2}$ groups, the rats were administered a dose of cisplatin on the 1st and 7 th days. The rats in the $\mathrm{Con}+\mathrm{H}_{2}$ and $\mathrm{OI}+\mathrm{H}_{2}$ groups were intraperitoneally injected with hydrogen-rich saline $(10 \mathrm{ml} / \mathrm{kg}$ body weight) once a day over a 2 -week period. On the 14 th, 28th and 42nd days $\left(\mathrm{T}_{1}, \mathrm{~T}_{2}\right.$ and $\left.\mathrm{T}_{3}\right)$ after the cisplatin injection, femoral vein blood was collected. At the end of the experiment, ovarian homogenates were prepared, and the samples were used for estrogen $\left(\mathrm{E}_{2}\right)$, follicle-stimulating hormone (FSH), superoxide dismutase (SOD), catalase (CAT) and malondialdehyde (MDA) examination. In addition, rats ( $\mathrm{n}=10$ per group) were sacrificed for bilateral ovary removal; one was fixed in formalin for follicle-counting analysis, while the other was used
\end{abstract}

Correspondence to: Dr Yonghao Yu or Dr Keliang Xie, Department of Anesthesiology, Tianjin Institute of Anesthesiology, Tianjin Medical University General Hospital, 406 Jiefang South Road, Tianjin 300052, P.R. China

E-mail: yuhonghao@126.com

E-mail: xiekeliang2009@hotmail.com

*Contributed equally

Abbreviations: CAT, catalase; $\mathrm{E}_{2}$, estrogen; ELISA, enzyme-linked immunosorbent assay; FSH, follicle stimulating hormone; MDA, malondialdehyde; Nrf2, nuclear factor erythroid 2-related factor 2; ROS, reactive oxygen species; SOD, superoxide dismutase

Key words: hydrogen-rich saline, cisplatin, ovarian injury, oxidative stress for nuclear factor erythroid 2-related factor 2 (Nrf2) detection by western blotting. Hydrogen-rich saline attenuated the FSH release, elevated the level of $\mathrm{E}_{2}$, improved the development of follicles, and reduced the damage to the ovarian cortex at $\mathrm{T}_{1}$, $\mathrm{T}_{2}$ and $\mathrm{T}_{3}$ in the $\mathrm{OI}+\mathrm{H}_{2}$ rats. Cisplatin induced oxidative stress by increasing the levels of oxidation products and attenuating the activity of antioxidant enzyme, which could be reversed by hydrogen-rich saline treatment. Furthermore, hydrogen-rich saline regulated the $\mathrm{Nrf} 2$ protein expression in rats with ovarian damage. In conclusion, hydrogen-rich saline exerts a protective effect against cisplatin-induced ovarian injury by reducing MDA and increasing SOD and CAT activity. Ovarian injury induced by chemotherapy involves the activation of $\mathrm{Nrf} 2$.

\section{Introduction}

In excess of 678,000 women were diagnosed with cancer in 2007 (1). A total of $2 \%$ of the cancer patients were $<40$ years old and at a prereproductive or reproductive age (1). Although effective chemotherapeutics and advanced treatment technology have increased the chances of long-term survival in cancer patients and improved the quality of life, the side effects of cancer treatments remain a neglected problem. While chemotherapeutics provide an effective cure method for female cancer patients aged $<40$ years, they can also induce damage to the ovarian reserve function (2).

Cisplatin (cis-diamine-dichloroplatinum II) is a chemotherapy drug that is commonly and widely used for the treatment of several types of cancer, including ovarian, breast and endometrial cancer (3-5); however, while cisplatin exerts a therapeutic effect in various types of cancer, its side effects also receive considerable attention. The side effects induced by cisplatin are associated with an increased production of free radicals and reactive oxygen species (ROS), leading to oxidative stress and lipoperoxidation (6). It is reported that cisplatin can cause oocyte and granulosa cell injury, as well as ovarian reserve insufficiency (7). Since ROS are closely associated with ovarian failure, the abnormal production of ROS may be involved in chemotherapy-induced ovarian damage.

Hydrogen has been reported to exert a therapeutic antioxidant effect, by selectively reducing cytotoxic ROS, and reduce levels of inflammation and apoptosis in several diseases (8-13). Furthermore, hydrogen may alleviate the nephrotoxicity 
induced by chemotherapy drugs without compromising their anti-tumor activity (12). Our previous study demonstrated that hydrogen treatment alleviated chemotherapy-induced ototoxicity by reducing oxidative stress (10); the aim of the present study was to investigate effect of hydrogen-rich saline treatment on chemotherapy-induced ovarian damage.

\section{Materials and methods}

Experimental animals. Adult, virgin, female Sprague Dawley rats, weighing 180-220 g, were obtained from the Laboratory Animal Center of the Academy of Military Medical Sciences (Beijing, China). The animals were housed at $20-22^{\circ} \mathrm{C}$ with a 12-h light/dark cycle and fed standard chow and water ad libitum. The procedures in this study were approved by the Animal Care and Use Committee of Tianjin Medical University (Tianjin, China) and performed in accordance with the guidelines for the use of experimental animals from the National Institutes of Health. Vaginal smears had been obtained daily for $>10$ days to manifest $\geq 2$ sequential, normal, 5 -day vaginal estrus cycles, in order to ensure the availability of experimental animals in this study

Hydrogen-rich saline production. The hydrogen-rich saline was prepared as previously described (13). Hydrogen was dissolved in physiological saline for $4 \mathrm{~h}$ under $0.4 \mathrm{MPa}$ pressure to a saturated level using a self-designed, hydrogen-rich, water-producing apparatus, which was stored under atmospheric pressure at $4^{\circ} \mathrm{C}$ in an aluminum bag with no dead volume. To ensure a concentration of $>0.6 \mathrm{mmol} / \mathrm{l}$, it was necessary to freshly prepare the hydrogen-rich saline every week. A needle-type hydrogen sensor (Unisense A/S, Aarhus, Denmark) was used to confirm the content of hydrogen in the saline (14).

Animal model establishment and grouping. A total of 240 animals were randomly divided into four groups $(n=60$ per group): Control (Con), control + hydrogen-rich saline $\left(\mathrm{Con}+\mathrm{H}_{2}\right)$, cisplatin-induced ovarian injury (OI) and cisplatin-induced ovarian injury + hydrogen-rich saline $\left(\mathrm{OI}+\mathrm{H}_{2}\right)$. The animal model was established using a previously described method (15) with little change. Cisplatin was diluted in saline immediately before use. In the $\mathrm{OI}$ and $\mathrm{OI}+\mathrm{H}_{2}$ groups, the rats received a single dose of cisplatin $[5 \mathrm{mg} / \mathrm{kg}$; the lethal dose- 50 of cisplatin in rats is $7.4 \mathrm{mg} / \mathrm{kg}$ (16)] by intraperitoneal injection on the 1st day. On the 7th day, the rats received another dose of cisplatin in an identical manner, which established the rat models of cisplatin-induced ovarian damage. An identical dose of saline was injected in the Con and Con $+\mathrm{H}_{2}$ group rats using the same method.

In the Con $+\mathrm{H}_{2}$ and $\mathrm{OI}+\mathrm{H}_{2}$ groups, the rats were intraperitoneally injected with hydrogen-rich saline $(10 \mathrm{ml} / \mathrm{kg}$ body weight) once a day between the 1st and the 14th day. Vaginal smears were obtained from each rat at 8:00 a.m. each day for $\geq 5$ days after cisplatin injection to ensure the ovarian injury model was successful.

Specimen collection. On the 14 th, 28 th and 42 nd days $\left(\mathrm{T}_{1}\right.$, $\mathrm{T}_{2}$ and $\mathrm{T}_{3}$ ) after cisplatin injection, femoral vein blood was collected from the rats ( $\mathrm{n}=10$ per group) and centrifuged at
$10,000 \times \mathrm{g}, 4^{\circ} \mathrm{C}$ for $10 \mathrm{~min}$. At the same time, ovarian tissue was collected, and homogenates were prepared via centrifugation, using the aforementioned conditions. These sample were stored at $-80^{\circ} \mathrm{C}$ until the estrogen $\left(\mathrm{E}_{2}\right)$, follicle-stimulating hormone (FSH), superoxide dismutase (SOD), catalase (CAT) and malondialdehyde (MDA) examination. At each time-point, another 10 rats ( $\mathrm{n}=10$ per group) were sacrificed for bilateral ovary removal; one was fixed in formalin for follicle-counting analysis, while the other was used for nuclear factor erythroid 2-related factor 2 (Nrf2) detection by western blotting.

Staining and ovarian follicle-counting analysis. Ovarian follicle populations were counted using previously described methods (15). The ovaries were fixed in 10\% formalin for $6 \mathrm{~h}$ at room temperature, embedded in paraffin and sectioned at a $5-\mu \mathrm{m}$ thickness. Following deparaffinization and rehydration, the sections were stained with hematoxylin and eosin. The oocyte-containing follicles in the developmental stage were classified, and the antral follicles were counted in every 12th section by two experienced pathologists. Follicle classification and counting was carried out according to the criteria of Oktay et al (17).

Detection of $E_{2}$ and FSH by enzyme-linked immunosorbent assay (ELISA). The serum $\mathrm{E}_{2}$ and FSH levels were measured using ELISA kits from R\&D Systems (Minneapolis, MN, USA). The assays were performed according to the manufacturer's instructions. The absorbance was read on a microplate reader (Denley Dragon Wellscan MK 3; Thermo Fisher Scientific, Vantaa, Finland), and the concentrations were calculated based on a standard curve. All standards and samples were run in duplicate.

Detection of antioxidant enzymes (SOD and CAT) and oxidation products (MDA). To investigate the mechanism associated with the effects of hydrogen-rich saline, the levels of antioxidant enzymes and oxidation products in the serum and ovarian tissue were measured. The activities of SOD, CAT and MDA were measured using commercial kits purchased from Cayman Chemical Company (Ann Arbor, MI, USA). According to the manufacturer's instructions, total SOD activity was assayed at $450 \mathrm{~nm}$ and CAT at $540 \mathrm{~nm}$. Spectrophotometric readings of SOD and CAT were performed using a DU-640B spectrophotometer (Beckman Coulter, Miami, FL, USA), while readings for MDA were obtained using a microplate reader (CA94089; Molecular Devices, Sunnyvale, CA, USA). All standards and samples were run in duplicate.

Detection of Nrf2 by western blotting. At each time-point, ovarian tissue was collected to lyse on ice in $100 \mu 1$ radioimmunoprecipitation assay buffer $[50 \mathrm{mmol} / 1$ Tris- $\mathrm{HCl}(\mathrm{pH} 7.4)$, $150 \mathrm{mmol} / 1 \mathrm{NaCl}, 1 \mathrm{mmol} / \mathrm{l}$ phenylmethanesulfonyl fluoride, $1 \mathrm{mmol} / 1$ ethylenediaminetetraacetic acid, $1 \%$ Triton X-100, $0.5 \%$ sodium deoxycholate and $0.1 \%$ sodium dodecyl sulfate]. The lysates were cleared by centrifugation at $15,000 \mathrm{x}$ g for $10 \mathrm{~min}$, and the supernatant protein samples were denatured at $100^{\circ} \mathrm{C}$ for $5 \mathrm{~min}$, separated on $10 \%$ acrylamide gels and then electrotransferred to polyvinylidene fluoride membranes. 

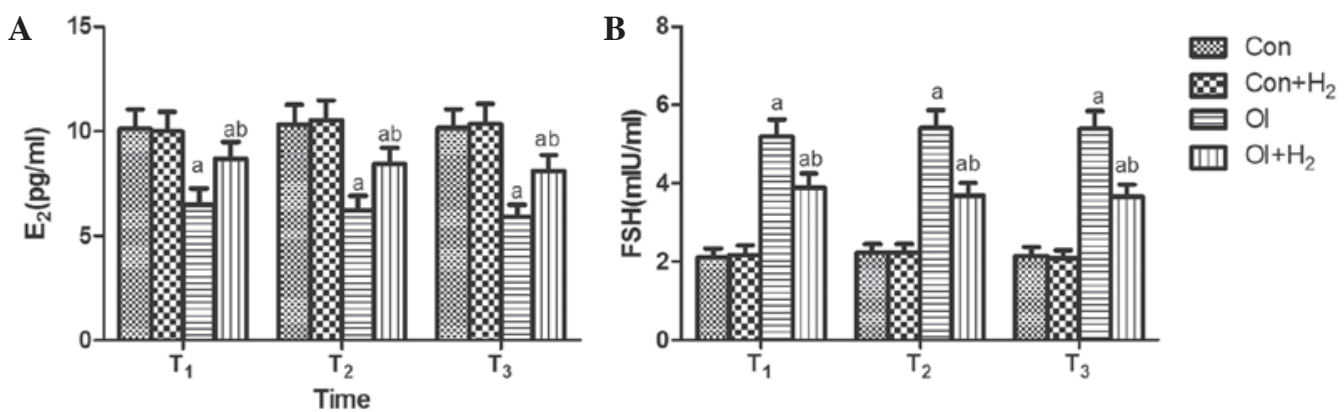

Figure 1. Time-dependent effects of hydrogen-rich saline on the release of sex hormones ( $\mathrm{E}_{2}$ and FSH) during the process of chemotherapy-induced ovarian injury. Cisplatin was injected twice, each time at $5 \mathrm{mg} / \mathrm{kg}$, in group OI. The same dose of saline was injected in group Con. The rats received intraperitoneal hydrogen-rich saline at $10 \mathrm{ml} / \mathrm{kg}$ body weight for 14 days. The levels of (A) FSH and (B) $\mathrm{E}_{2}$ were measured on the 14 th, 28th and $42 \mathrm{nd} \mathrm{days} \mathrm{(} \mathrm{T}_{1}$, $\mathrm{T}_{2}$ and $\mathrm{T}_{3}$ ) after the cisplatin injection. Results are presented as the mean \pm standard deviation ( $\mathrm{n}=10$ per group). ${ }^{\text {a }} \mathrm{P}<0.05$ compared with group Con; ${ }^{\text {b }} \mathrm{P}<0.05$ compared with group OI. $\mathrm{E}_{2}$, estrogen; FSH, follicle-stimulating hormone; Con, control; Con $+\mathrm{H}_{2}$, control + hydrogen-rich saline; OI, cisplatin-induced ovarian injury; $\mathrm{OI}+\mathrm{H}_{2}$, cisplatin-induced ovarian injury + hydrogen-rich saline.

Following the blocking of the membranes with $5 \%$ non-fat dried milk in Tris buffer saline tween 20 buffer at room temperature for $2 \mathrm{~h}$, primary rabbit polyclonal antibodies against Nrf2 (1:200; ab92946; Abcam, Cambridge, MA,USA) and $\beta$-actin $(1: 2,000 ;$ ab129348; Abcam) were added for incubation overnight at $4^{\circ} \mathrm{C}$. The immunoblots were subsequently incubated with horseradish peroxidase-conjugated goat anti-rabbit IgG (ab175773; Abcam, Cambridge, MA,USA and detected using enhanced chemiluminescence reagent (Merck Millipore, Molsheim, France). Images of the immunoblots were visualized and captured using the Quantity One ${ }^{\circledR}$ quantitative gel analysis system (Bio-Rad, Tokyo, Japan). All western blot analyses were carried out $\geq 3$ times.

Statistical analysis. Differences between the groups were analyzed using one-way analysis of variance followed by the Tukey comparison. Results are expressed as the mean \pm standard deviation of $\geq 3$ independent experiments, and $\mathrm{P}<0.05$ was considered to indicate a statistically significant difference between groups.

\section{Results}

Hydrogen-rich saline regulates $E_{2}$ and FSH during the process of chemotherapy-induced ovarian injury. Following the injection of two single doses of $5 \mathrm{mg} / \mathrm{kg}$ cisplatin in the rats, abnormal levels of the sex hormones appeared in the serum. Compared with group Con, no significant difference in the release of FSH was found in group Con $+\mathrm{H}_{2}(\mathrm{P}>0.05$, Fig. 1); however, the release of FSH was increased and $\mathrm{E}_{2}$ release was reduced in group $\mathrm{OI}(\mathrm{P}<0.05$, Fig. 1$)$. The administration of hydrogen-rich saline (10 $\mathrm{ml} / \mathrm{kg}$ body weight) for 2 weeks during cisplatin injection attenuated the FSH release and elevated the level of $\mathrm{E}_{2}$ at $\mathrm{T}_{1}, \mathrm{~T}_{2}$ and $\mathrm{T}_{3}$ compared with group OI ( $\mathrm{P}<0.05$, Fig. 1).

Effect of hydrogen-rich saline ondifferent developmental stages of the follicle during the process of chemotherapy-induced ovarian injury in rats. All developmental stages of the follicles, and the structure of the ovarian cortex and medulla, could be clearly seen in group Con. Compared with group Con, few follicles were observed in group OI; furthermore, the group OI rats exhibited significant damage to the cortex, which could not be distinguished from the medulla. Compared with group $\mathrm{OI}$, the development of the follicles in group $\mathrm{OI}+\mathrm{H}_{2}$ was much more regular; all developmental stages of the follicles could be seen, and the damage to the cortex was less severe $(\mathrm{P}<0.05$, Fig. 2).

Effect of hydrogen-rich saline on antioxidant enzymes (SOD and $C A T$ ) and the oxidation product MDA during chemotherapy in rats. To determine the underlying mechanism of the effects observed, antioxidant enzymes and oxidation products were detected in the serum and ovarian tissue of the rats at the $T_{1}, T_{2}$ and $T_{3}$ time-points. As shown in Fig. 3, when compared with group Con, cisplatin induced an excessive release of MDA and inhibited the activity of SOD and CAT in the serum and ovarian tissue of group OI $(\mathrm{P}<0.05)$. Hydrogen-rich saline significantly reduced the levels of MDA and elevated the activity of SOD and CAT in the serum and ovarian tissue of the cisplatin-challenged rats $(\mathrm{P}<0.05)$. The results revealed that cisplatin led to oxidative stress by increasing the levels of oxidation products and attenuating the activity of antioxidant enzymes, which could be reversed by hydrogen-rich saline treatment.

Hydrogen-rich saline elevates Nrf2 expression in the ovarian tissue of the chemotherapy-induced rats. Nrf2 is the key molecule of the Nrf2/antioxidant response element (ARE) signaling pathway, which is a relatively conservative and important endogenous antioxidative system (18). Cisplatin induced $\mathrm{Nrf} 2$ expression in the ovarian tissue of the rats between $\mathrm{T}_{1}$ and $\mathrm{T}_{3}(\mathrm{P}<0.05$, Fig. 4). Compared with group OI, hydrogen-rich saline treatment further increased the Nrf2 expression between $T_{1}$ and $T_{3}$. Based on the results, it was suggested that hydrogen-rich saline may regulate the Nrf2/ARE signaling pathway to protect cells from immoderate oxidative stress.

\section{Discussion}

In the present study, cisplatin stimulation was used to establish a model of ovarian injury in young female rats. Compared with group OI, it was found that hydrogen-rich 


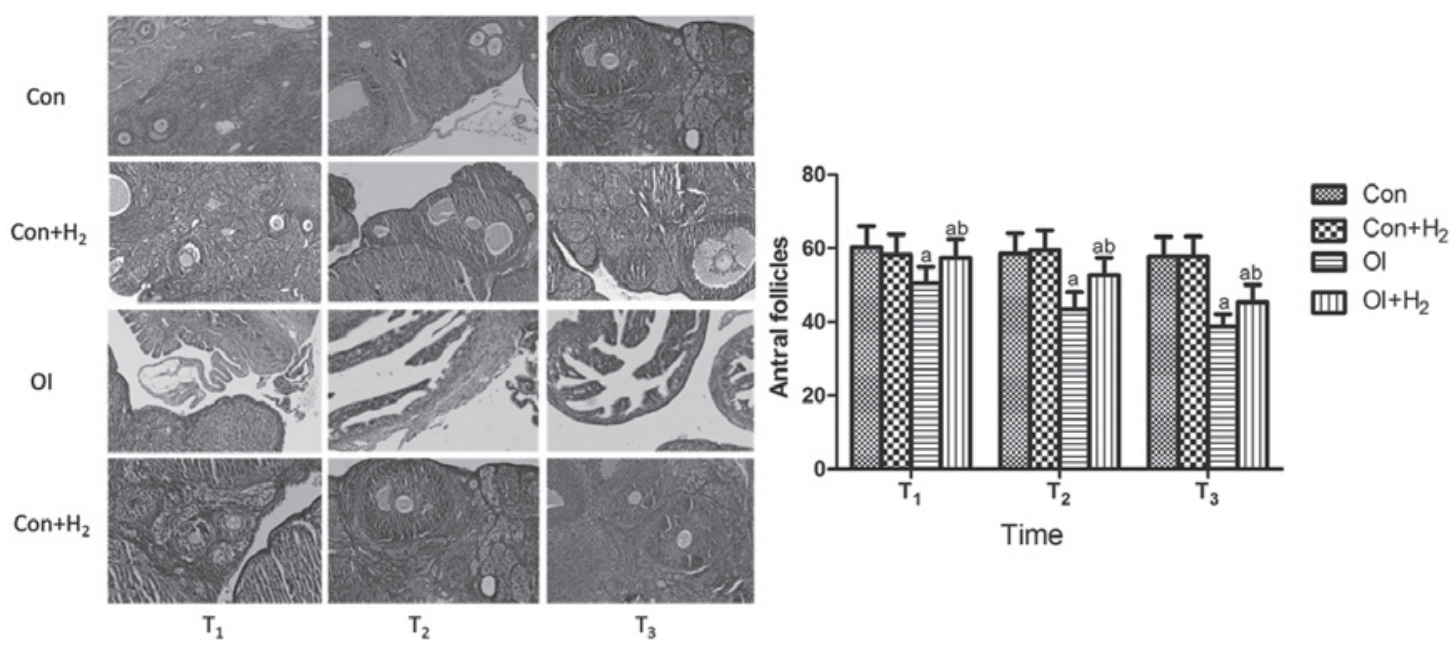

Figure 2. Effect of hydrogen-rich saline on the different developmental stages of follicles during the process of chemotherapy-induced ovarian injury in rats (hematein eosin staining; magnification, x100). Cisplatin was injected at $5 \mathrm{mg} / \mathrm{kg}$ in group OI. Rats received intraperitoneal hydrogen-rich saline at $10 \mathrm{ml} / \mathrm{kg}$ body weight for 14 days. The number of antral follicles was counted on the 14th, 28th and 42nd days $\left(\mathrm{T}_{1}, \mathrm{~T}_{2}\right.$ and $\left.\mathrm{T}_{3}\right)$ after the cisplatin injection. All levels of the follicles, as well as the clear structure of the cortex, could be observed in group Con. Few follicles could be seen in group OI. In group OI $+\mathrm{H}_{2}$ the development of the follicles was much more regular, all levels of follicles could be determined and the damage to the cortex was less severe. Results are presented as the mean \pm standard deviation ( $\mathrm{n}=10$ per group). ${ }^{\mathrm{a}} \mathrm{P}<0.05$ compared with group Con; ${ }^{\mathrm{b}} \mathrm{P}<0.05$ compared with group OI. Con, control; Con $+\mathrm{H}_{2}$, control + hydrogen-rich saline; OI, cisplatin-induced ovarian injury; $\mathrm{OI}+\mathrm{H}_{2}$, cisplatin-induced ovarian injury + hydrogen-rich saline.

A

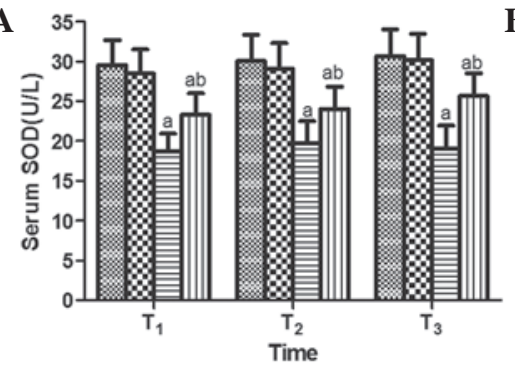

D

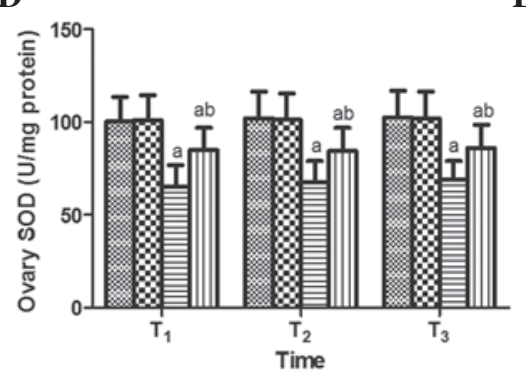

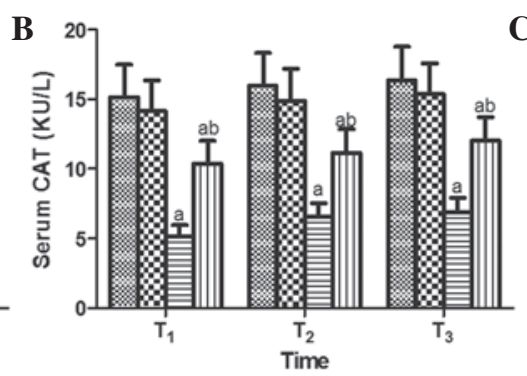
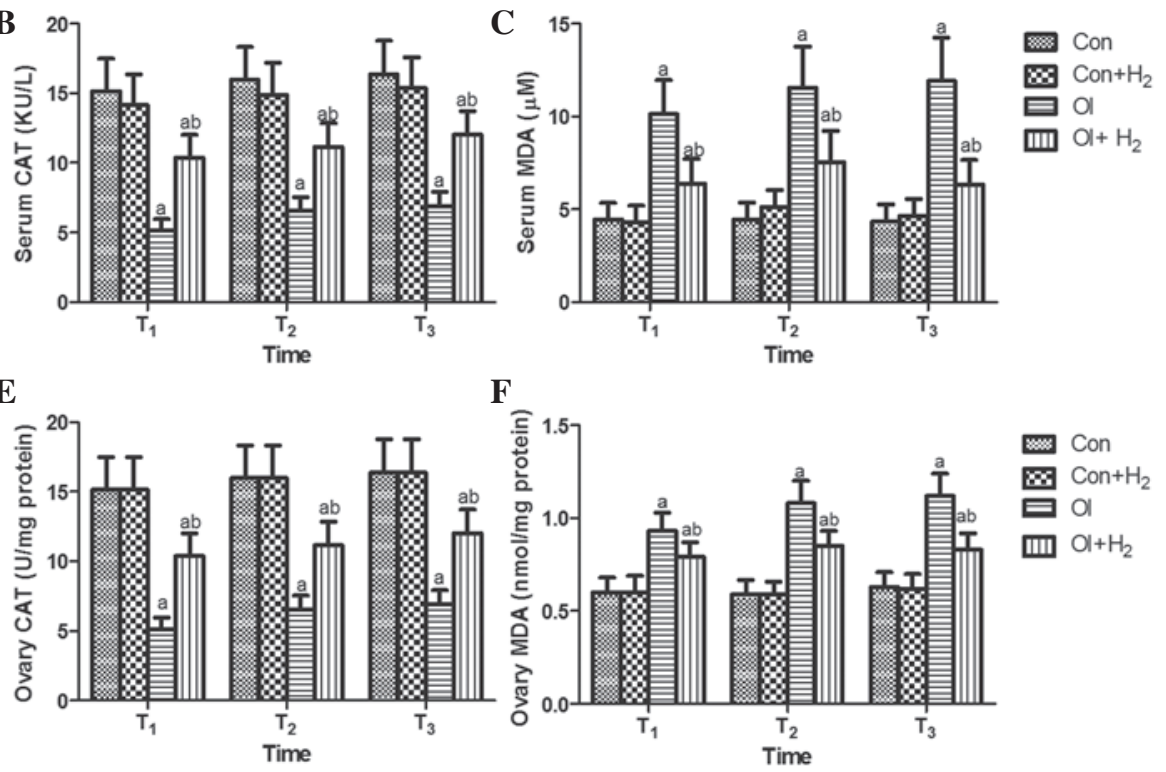

Figure 3. Hydrogen-rich saline regulates cisplatin-induced oxidative stress by increasing the activity of SOD and CAT and reducing the level of MDA in the serum and ovarian tissue of rats. Cisplatin was injected at $5 \mathrm{mg} / \mathrm{kg}$ in group OI. Rats received intraperitoneal hydrogen-rich saline at $10 \mathrm{ml} / \mathrm{kg}$ body weight for 14 days. On the 14th, 28th and 42nd days $\left(T_{1}, T_{2}\right.$ and $\left.T_{3}\right)$ after the cisplatin injection, the (A-C) blood and (D-F) tissue samples were collected for the measurement of (A and D) SOD, (B and E) CAT and (C and F) MDA. Values are expressed as the mean \pm standard deviation ( $\mathrm{n}=10$ per group). ${ }^{\text {a }}<0.05$ compared with group Con; ${ }^{b} \mathrm{P}<0.05$ compared with group OI. SOD, superoxide dismutase; CAT, catalase; MDA, malondialdehyde; Con, control; Con $+\mathrm{H}_{2}$, control + hydrogen-rich saline; $\mathrm{OI}$, cisplatin-induced ovarian injury; $\mathrm{OI}+\mathrm{H}_{2}$, cisplatin-induced ovarian injury + hydrogen-rich saline.

saline treatment regulated the release of sex hormones and markedly improved the pathological condition and antral follicle counts. Hydrogen-rich saline additionally improved the production of antioxidant enzymes and attenuated the levels of oxidation products following cisplatin injection. These results indicate that hydrogen-rich saline treatment exerts a protective effect against ovarian injury in rats by regulating the balance of the redox system.

Cisplatin is a commonly used chemotherapeutic agent that exerts a therapeutic effect in various types of cancer and is often used to treat female patients at a reproductive age; however, despite its therapeutic effect, the side effects of cisplatin have also received considerable attention. The side effects of cisplatin are associated with an excessive production of free radicals and ROS, such as superoxide anions or $\mathrm{H}_{2} \mathrm{O}_{2}$, in different kidney cells (19) and in ovarian cancer $(6,20)$. To investigate the side effects of cisplatin on the ovaries in the present study, an intraperitoneal injection of cisplatin was used to induce ovarian injury in female rats of a reproductive age. The results revealed that cisplatin 

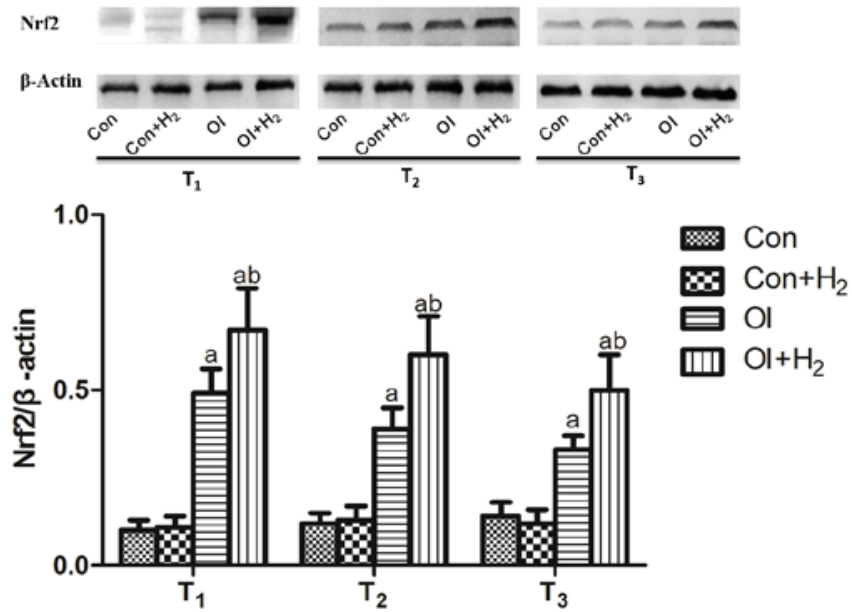

Figure 4. Hydrogen-rich saline increases Nrf2 expression in ovarian tissue on the 14th, 28th and 42nd days $\left(\mathrm{T}_{1}, \mathrm{~T}_{2}\right.$ and $\left.\mathrm{T}_{3}\right)$ after cisplatin injection. Ovarian tissue was collected to detect the Nrf2 and $\beta$-actin expression using western blotting. Values are expressed as the mean \pm standard deviation $(n=10$ per group). ${ }^{\text {a }}<0.05$ compared with group $\mathrm{Con} ;{ }^{\text {b }} \mathrm{P}<0.05$ compared with group OI. Nrf2, nuclear factor erythroid 2-related factor 2; Con, control; Con $+\mathrm{H}_{2}$ control + hydrogen-rich saline; $\mathrm{OI}$, cisplatin-induced ovarian injury; $\mathrm{OI}+\mathrm{H}_{2}$, cisplatin-induced ovarian injury + hydrogen-rich saline.

attenuated $\mathrm{E}_{2}$ secretion, increased FSH secretion by negative feedback and damaged the structure of the cortex in female rats with ovarian injury.

It has previously been found that hydrogen exerts protective effects against various diseases. Hydrogen selectively alleviates hydroxyl radicals, other ROS and oxidation products and has been shown to improve the activity of antioxidants in a number of disease models, including shock, multiple organ dysfunction syndrome and ischemia-reperfusion (8-13). In addition, hydrogen exerts a protective effect against chemotherapy-induced organ injury, and studies using dynamic contrast-enhanced computed tomography (19) and blood oxygenation level-dependent magnetic resonance imaging (11) have demonstrated that hydrogen-rich water protects the kidneys against cisplatin-induced nephrotoxicity without compromising the anti-tumor activity (12). Hydrogen additionally exerts an antitumor effect. It has been reported that hydrogen reduced the size of skin tumors of squamous cell carcinoma in hairless albino mice (21), and platinum nanocolloid-supplemented hydrogen-rich water inhibited the clonal growth of human tongue carcinoma cells (22). These studies suggest that hydrogen has the potential ability to protect ovarian tissue from cisplatin-induced injury and may exert an antitumor effect in cancer patients. In the present study, hydrogen-rich saline treatment increased $\mathrm{E}_{2}$ secretion and reduced FSH secretion in ovarian injury rats. The saline also had a protective effect against damaged cells and tissue, either by slowing the cell damage process or activating certain signaling pathways in the cells.

It has previously been shown that the follicle microenvironment consists of a dynamic balance of oxidation and antioxidation, including antioxidant enzymes in the follicular fluid (such as SOD and CAT), which has a close association with follicle maturation (15). High levels of oxidation products (MDA) and/or a decreased antioxidative ability in oocytes (such as low SOD activity) may, therefore, disrupt the redox system balance and lead to oxidative stress. Oxidative stress can induce the blockade of follicular development and ovulation and result in menstrual disorder (amenorrhea, oligomenorrhea) and even infertility (23-26). SOD as an antioxidant enzyme can eliminate oxygen radicals by accelerating the dismutation of superoxide to $\mathrm{H}_{2} \mathrm{O}_{2}$, and CAT catalyzes the degradation of $\mathrm{H}_{2} \mathrm{O}_{2}$ into $\mathrm{H}_{2} \mathrm{O}$ and $\mathrm{O}_{2}$. SOD and CAT therefore act as mutually supportive antioxidative enzymes that actively defend against ROS (27). Matzuk et al (28) reported that sub-fertile or infertile SOD1-knockout mice had ovarian defects and suppressed FSH and luteinizing hormone levels; therefore, SOD has a vital role in ovarian injury. The present results showed that cisplatin induced an excessive production of MDA and reduced the SOD and CAT activity. Hydrogen treatment reversed the effect of cisplatin on MDA, SOD and CAT, in addition to regulating the sex hormone secretion.

Nrf2, as a member of the cap'n'collar basic leucine zipper subfamily of leucine zipper transcription factors, is one of the key factors involved in initiating the endogenous protective effect against oxidative stress. Following stimulation by chemical toxicity, carcinogenesis and pathological processes, Nrf2 and its cytoplasmic binding protein, Kelch-like ECH-associated protein 1, become uncoupled, and Nrf2 is transferred into the nucleus. Nrf2 combines with ARE to induce two sets of target genes: Phase II detoxification enzymes and antioxidant enzymes, which play an essential role in cellular protection $(29,30)$. Hu et al (31) previously demonstrated that Nrf2 serves as an essential sensor and regulator of chemical homeostasis in ovarian cells, protecting the cells from toxic chemicals by controlling metabolic detoxification, ROS defense and forkhead box protein $\mathrm{O} 3$ expression. Without Nrf2 expression in the mice, the host became more sensitive to harmful stimulation, facilitating organ injury (31). In the present study it was found that Nrf2 expression was increased following the use of cisplatin to induce a model of ovarian injury in rats, and this may have been due to Nrf2 acting as a stress response following stimulation by chemotherapeutic agents. Hydrogen-rich saline treatment further increased the Nrf2 expression in the chemotherapy-induced ovarian injury rats.

There were several limitations in the present study. First, the changes in oxidation product and antioxidative enzymes were measured in vivo; the expression levels and changes should therefore be verified in vitro using ovarian cells. Secondly, the role of Nrf2 should be more comprehensively investigated, using an inhibitor of Nrf2 or Nrf2-knockdown rats; this will be the aim of our next experiment.

In conclusion, exposure to certain harmful factors can lead to the destruction of ovarian follicles in animal models. Cisplatin induces abnormal sex hormone secretion in a rat model of ovarian injury. Hydrogen-rich saline exerts a protective effect against cisplatin-induced ovarian injury by reducing MDA and increasing SOD and CAT activity. Furthermore, high Nrf2 expression is associated with ovarian injury.

\section{Acknowledgements}

This study was supported by the National Natural Science Foundation of China (grant nos. 81372033 and 81101409), the 
Natural Science Foundation of the Tianjin Science Committee (grant nos. 11JCYBJC12900 and 13JCQNJC11400) and the Foundation of Tianjin Bureau of Public Health (grant no. 2011KZ108).

\section{References}

1. Jemal A, Siegel R, Ward E, Murray T, Xu J and Thun MJ: Cancer statistics, 2007. CA Cancer J Clin 57: 43-66, 2007.

2. Lee HJ, Selesniemi K, Niikura Y, Niikura T, Klein R, Dombkowski DM and Tilly JL: Bone marrow transplantation generates immature oocytes and rescues long-term fertility in a preclinical mouse model of chemotherapy-induced premature ovarian failure. J Clin Oncol 25: 3198-3204, 2007.

3. Zhang Y, Cheng Y, Ren X, Zhang L, Yap KL, Wu H, Patel R, Liu D, Qin ZH, Shih IM and Yang JM: NAC1 modulates sensitivity of ovarian cancer cells to cisplatin by altering the HMGB1-mediated autophagic response. Oncogene 31: 1055-1064, 2012.

4. Silver DP, Richardson AL, Eklund AC, Wang ZC, Szallasi Z, Li Q, Juul N, Leong CO, Calogrias D, Buraimoh A, et al: Efficacy of neoadjuvant Cisplatin in triple-negative breast cancer. J Clin Oncol 28: 1145-1153, 2010.

5. Bae-Jump VL, Zhou C, Boggess JF and Gehrig PA: Synergistic effect of rapamycin and cisplatin in endometrial cancer cells. Cancer 115: 3887-3896, 2009.

6. Hu J and Friedman E: Depleting Mirk kinase increases cisplatin toxicity in ovarian cancer cells. Genes Cancer 1: 803-811, 2010.

7. Morgan S, Lopes F, Gourley C, Anderson RA and Spears N: Cisplatin and doxorubicin induce distinct mechanisms of ovarian follicle loss; imatinib provides selective protection only against cisplatin. PLoS One 8: e70117, 2013.

8. Xie K, Yu Y, Pei Y, Hou L, Chen S, Xiong L and Wang G: Protective effects of hydrogen gas on murine polymicrobial sepsis via reducing oxidative stress and HMGB1 release. Shock 34: 90-97, 2010.

9. Xie K, Yu Y, Zhang Z, Liu W, Pei Y, Xiong L, Hou L and Wang G: Hydrogen gas improves survival rate and organ damage in zymosan-induced generalized inflammation model. Shock 34 : 495-501, 2010.

10. Qu J, Li X, Wang J, Mi W, Xie K and Qiu J: Inhalation of hydrogen gas attenuates cisplatin-induced ototoxicity via reducing oxidative stress. Int J Pediatr Otorhinolaryngol 76: 111-115, 2012.

11. Matsushita T, Kusakabe Y, Kitamura A, Okada S and Murase K: Investigation of protective effect of hydrogen-rich water against cisplatin-induced nephrotoxicity in rats using blood oxygenation level-dependent magnetic resonance imaging. Jpn J Radiol 29: 503-512, 2011.

12. Nakashima-Kamimura N, Mori T, Ohsawa I, Asoh S and Ohta S: Molecular hydrogen alleviates nephrotoxicity induced by an anti-cancer drug cisplatin without compromising anti-tumor activity in mice. Cancer Chemother Pharmacol 64: 753-761, 2009.

13. Zheng X, Mao Y, Cai J, Li Y, Liu W, Sun P, Zhang JH, Sun X and Yuan H: Hydrogen-rich saline protects against intestinal ischemia/reperfusion injury in rats. Free Radic Res 43: 478-484 2009.

14. Hayashida K, Sano M, Ohsawa I, Shinmura K, Tamaki K, Kimura K, Endo J, Katayama T, Kawamura A, Kohsaka S, et al: Inhalation of hydrogen gas reduces infarct size in the rat model of myocardial ischemia-reperfusion injury. Biochem Biophys Res Commun 373: 30-35, 2008.
15. Li X, Yang S, Lv X, Sun H, Weng J, Liang Y and Zhou D: The mechanism of mesna in protection from cisplatin-induced ovarian damage in female rats. J Gynecol Oncol 24: 177-185, 2013.

16. Dale O, Mortensen B, Thommesen L and Hagen B: Cisplatin toxicity in the rat may be influenced by anaesthetic agents. Acta Anaesthesiol Scand 44: 770, 2000.

17. Oktay K, Schenken RS and Nelson JF: Proliferating cell nuclear antigen marks the initiation of follicular growth in the rat. Biol Reprod 53: 295-301, 1995.

18. Cheng X, Ku CH, Siow RC. Regulation of the Nrf2 antioxidant pathway by microRNAs: New players in micromanaging redox homeostasis. Free Radic Biol Med 64: 4-11, 2013.

19. Li Y, Li X, Wong YS, Chen T, Zhang H, Liu C and Zheng W: The reversal of cisplatin-induced nephrotoxicity by selenium nanoparticles functionalized with 11-mercapto-1-undecanol by inhibition of ROS-mediated apoptosis. Biomaterials 32: 9068-9076, 2011.

20. Belotte J, Fletcher NM, Awonuga AO, Alexis M, Abu-Soud HM, Saed MG, Diamond MP and Saed GM: The role of oxidative stress in the development of cisplatin resistance in epithelial ovarian cancer. Reprod Sci 21: 503-508, 2014.

21. Dole M, Wilson FR and Fife WP: Hyperbaric hydrogen therapy: A possible treatment for cancer. Science 190: 152-154, 1975.

22. Saitoh Y, Yoshimura Y, Nakano K and Miwa N: Platinum nanocolloid-supplemented hydrogen-dissolved water inhibits growth of human tongue carcinoma cells preferentially over normal cells. Exp Oncol 31: 156-162, 2009.

23. Oyawoye O, Abdel Gadir A, Garner A, Constantinovici N, Perrett $C$ and Hardiman P: Antioxidants and reactive oxygen species in follicular fluid of women undergoing IVF: Relationship to outcome. Hum Reprod 18: 2270-2274, 2003.

24. Crha I, Hrubá D, Ventruba P, Fiala J, Totusek J and Visnová H: Ascorbic acid and infertility treatment. Cent Eur J Public Health 11: 63-67, 2003.

25. Carbone MC, Tatone C, Delle Monache S, Marci R, Caserta D, Colonna R and Amicarelli F: Antioxidant enzymatic defences in human follicular fluid: Characterization and age-dependent changes. Mol Hum Reprod 9: 639-643, 2003.

26. Török A, Belágyi J, Török B, Tinneberg HR and Bódis J: Scavenger capacity of follicular fluid, decidua and culture medium with regard to assisted reproduction: An in vitro study using electron paramagnetic resonance spectroscopy. Gynecol Obstet Invest 55: 178-182, 2003.

27. Cohen M, Lippman M and Chabner B: Role of pineal gland in aetiology and treatment of breast cancer. Lancet 2: 814-816, 1978.

28. Matzuk MM, Dionne L, Guo Q, Kumar TR and Lebovitz RM: Ovarian function in superoxide dismutase 1 and 2 knockout mice. Endocrinology 139: 4008-4011, 1998.

29. Nguyen T, Yang CS and Pickett CB: The pathways and molecular mechanisms regulating Nrf2 activation in response to chemical stress. Free Radic Biol Med 37: 433-441, 2004.

30. Dinkova-Kostova AT, Holtzclaw WD, Cole RN, Itoh K, Wakabayashi N, Katoh Y, Yamamoto M and Talalay P: Direct evidence that sulfhydryl groups of Keap1 are the sensors regulating induction of phase 2 enzymes that protect against carcinogens and oxidants. Proc Natl Acad Sci USA 99: 11908-11913, 2002.

31. Hu X, Roberts JR, Apopa PL, Kan YW and Ma Q: Accelerated ovarian failure induced by 4 -vinyl cyclohexene diepoxide in Nrf2 null mice. Mol Cell Biol 26: 940-954, 2006. 